\title{
VIBRODIAGNOSTICS OF COMPRESSOR VALVES VIA MUSIC PSEUDO-SPECTRA
}

\author{
A.A. Khvostov, V.I. Ryazhskih, I.A. Kazmin, N.A. Degtyarev, A.V. Ivanov \\ MESC AF N.E. Zhukovsky and Y.A. Gagarin Air Force Academy, Voronezh, Russia
}

\begin{abstract}
The paper presents a mathematical model of a piston compressor which is part of nitrogen-oxygen gas generator. Taking into account the structural parameters of the compressor, as well as the suction and pressurization valves, such model allows to obtain the dynamics of the pressure amplitude under the compressor's piston together with the vibration velocity and vibration acceleration that are transmitted in the form of mechanical affections to the equipment (such mechanical affections can be observed using the corresponding sensors). Also we investigate the pseudo-spectra of vibration signals using the MUSIC algorithm for the set of parameters of compressor's model (such parameters determine the evolution of a defect).
\end{abstract}

Keywords: vibrodiagnostics, piston compressors, nitrogen oxygen producing station, pseudospectrum, suction and delivery compressor valves.

Citation: Khvostov AA, Ryazhskih VI, Kazmin IA, Degtyarev NA, Ivanov AV. Vibrodiagnostics of Compressor Valves via MUSIC Pseudo-spectra. CEUR Workshop Proceedings, 2016; 1638: 588-592. DOI: 10.18287/16130073-2016-1638-588-592

\section{Introduction}

The analysis of operation of the Nitrogen-Oxygen Gas Generator (NOGG) has shown that one of the major reasons for stoppage is a fault in the high-pressure pneumatic systems. At the same time the main reason for failure in the high pressure pneumatic system is the failure of the valves in suction and pressurization lines.

In this way the quality of operation of the valves substantially determines the efficiency of compressor's operation as well as the NOGG operation. The defects of valves lead to an increase of energy expended for pushing of the gas, as well as to reducing the compressor efficiency together with the fact that the cost of air production at certain pressure grows [1]. The existing systems of vibration diagnostics use individual algorithms for detection of each kind of defect at the initial stage of its evolution. These algorithms allow to carry out the functional diagnostics for the objects at the nominal regime of the compressor's operation [2]. This allows to predict the technical condition of equipment and to prevent the station failure at a critical moment [3]. 
However, it is necessary to develop a mathematical model of pneumatic equipment taking into account the evolution of the probable major defects. This will allow to identify the main vibration features of the valve's failures. Also such a mathematical model will allow to construct the algorithms for information processing from the vibration sensors and to investigate the spectra of the recorded signals (this allows to make a decision about the system state via the set of vibration features of the defects) [4].

\section{Mathematical model of compressor}

In order to make a simulation of the evolution of defects of the valves in suction and pressurization lines we developed a mathematical model of the compressor taking into account the valve's defects evolution.

In this model of compressor we considered the following main functional elements of the system: compressor's piston which moves in the cylinder under affection of the crank mechanism; suction and pressurization valves of the compressor that are moving under the force caused by the difference of the pressure between the chamber under the piston and the suction line (or pressurization line); volume of the chamber under the piston which depends on the pressure.

The equation of motion of the piston (1) relative to the coordinate $x$ is constructed on the basis of the force balance (the compressor is placed in a horizontal position, the projection of gravity force on the $x$-axis is zero). The dynamics of $P_{\text {comp }}$ is described by the equation (2) which is based on the equation of state for an ideal gas with constant mass taking into account the isothermal conditions [5]. In order to make a qualitatively analysis of the real processes we use a simplified calculation scheme for selfacting valves [6,7]. In this scheme the valve is replaced with a conventional hole without friction and heat transfer losses. The gas flow in the valve is determined by the areas of the holes $S_{\text {valvel }}, S_{\text {valve2. }}$. For each valve we introduce a new coordinate system, namely $x_{1}\left(x_{2}\right)$ for suction (pressurization) valve. The motion of the plates of the valves with weights $m_{1}, m_{2}$ under the gravity force, viscous friction, spring reaction $c_{v a l 1}$ and inertia is described by the equations (3) and (5). The change of pressure in the chamber due to the expiration through the suction and pressurization valves is described by the equations (4), (6) that are obtained using the equation of state for an ideal gas, as well as the dependence (on the speed and hole's area) of the amount of gas flowing through the variable section $S_{\text {slice }}^{[1]}=x_{1} \pi D_{\text {valve }}, S_{\text {slice }}^{[2]}=x_{2} \pi D_{\text {valve2 }}$, where $D_{\text {valve1 }}, D_{\text {valve2 }}$ are the diameters of suction and pressurization valves, respectively. 


$$
\begin{aligned}
& \int m_{p} \frac{d^{2} x}{d t^{2}}+\mu_{f r} m_{p} g \operatorname{sign}\left(\frac{d x}{d t}\right)+S_{p} P_{c o m p}=F_{e x t} ; \\
& \frac{d P_{\text {comp }}}{d t}=\frac{P_{\text {comp }}+P_{r e l}^{[1]}-P_{r l}^{[2]}}{\left(H_{p}+H_{d v}-x\right)} \frac{d x}{d t} ; \\
& 0 \leq x \leq H_{p} \text {; } \\
& m_{\text {valvel }} \frac{d^{2} x_{1}}{d t^{2}}+\tau_{\text {valvel }} \frac{d x_{1}}{d t}+c_{\text {spr.valvel } 1} x_{1}=S_{\text {valvel }}\left(P_{\text {suc }}-P_{\text {comp }}\right) ; \\
& 0 \leq x_{1} \leq X_{k z 1} ; \\
& \left\{\frac{d P_{\text {rel }}^{[1]}}{d t}=\rho x_{1} \pi D_{\text {valve1 }} \frac{R T}{V^{[1]}} \sqrt{\frac{2\left|P_{\text {suc }}-P_{\text {comp }}\right|}{\xi^{[1]} \rho}} \operatorname{sign}\left(P_{\text {suc }}-P_{\text {comp }}\right) ;\right. \\
& m_{\text {valve } 2} \frac{d^{2} x_{2}}{d t^{2}}+\tau_{\text {valve } 2} \frac{d x_{2}}{d t}+c_{\text {spr.valve } 2} x_{2}=S_{\text {valve } 2}\left(P_{\text {del }}-P_{\text {comp }}\right) \text {; } \\
& 0 \leq x_{2} \leq X_{\text {valve2 }} \text {; } \\
& \frac{d P_{\text {rel }}^{[2]}}{d t}=\rho x_{2} \pi D_{\text {valve } 2} \frac{R T}{V^{[2]}} \sqrt{\frac{2\left|P_{\text {del }}-P_{\text {comp }}\right|}{\xi^{[2]} \rho}} \operatorname{sign}\left(P_{\text {del }}-P_{\text {comp }}\right) ; \\
& x(0)=x_{0}, \frac{d x}{d t}(t=0)=x_{v 0}, x_{1}(0)=x_{01}, \frac{d x_{1}}{d t}(t=0)=x_{v 01}, \\
& x_{2}(0)=x_{02}, \frac{d x_{2}}{d t}(t=0)=x_{v 02} \text {; } \\
& P_{\text {comp }}(0)=P_{\text {comp } 0}, P_{d e l}^{[1]}(0)=P_{d e l 0}^{[1]}, P_{d e l}^{[2]}(0)=P_{d e l 0}^{[2]} \text {. }
\end{aligned}
$$

Here $x$ is the coordinate, $t$ is the time, $m_{p}$ is a weight of the piston, $S_{p}$ is the area of the edge surface of the piston, $\operatorname{sign}(z)$ is the standard signum-function, $\mu_{f r}$ is the friction coefficient, $F_{\text {ext }}$ is the force affection from the crank mechanism, $P_{\text {comp }}$ is the pressure in the chamber under the piston, $H_{p}$ is the stroke, $H_{d v}$ is the displacement relative to the $x$-axis which corresponds to the "dead" volume of the chamber, $P_{d e l}^{[1]}, P_{d e l}^{[2]}$ are the pressure losses due to the air flow through the suction and pressurization valves, $\tau_{\text {valvel }}, \tau_{\text {valve } 2}$ are the damping coefficient of the valve (it depends on the constructive features of the valve, viscosity and density of a gas surrounding the plate), $\rho$ is the density of the gas, $\xi^{[1]}, \xi^{[2]}$ are the coefficients of hydraulic resistance of the valves, $X_{\text {valve2 }}, X_{\text {valvel }}$ are the maximum strokes of the valves, $P_{\text {suc }}$ is the pressure in suction line, $P_{d e l}$ is the pressure in pressurization line.

\section{$2 \quad$ Numerical simulation}

The mathematical model is realized in MathWorks Simulink ${ }^{\mathrm{TM}}$ simulation software. The numerical experiments included the following steps: 
1. Start the simulation process and recording the signals of pressure's amplitude together with the speed of its variation and corresponding acceleration.

2. Recording of the arrays of amplitudes, velocities, and accelerations as a gauge signal (this signal corresponds to the operation of healthy compressor) in the database (DB) of vibration signals.

3. Simulation of the valve's defect by changing the corresponding parameter in the mathematical model and start the process of simulation.

4. Calculation of the spectra of vibration signals from the DB for each of the simulated defects. Formation of DB of the vibration signals spectra.

5. Graphic and parametric representation of the obtained spectra and evaluation of significance of differences in the spectra due to the valve's defect.

6. Making decision on the possibility of diagnosis of the defect by the selected vibration features in the spectrum of the analyzed signal.

In order to construct the spectrum of the signals of pressure's amplitude, velocity and acceleration in the chamber under the piston of the compressor we use the MUSIC (MultipleSIgnal Classification) algorithm. It is designed for spectral analysis of the signals that are represented by the sum of multiple sine waves (multiple complex exponents in general case) with white noise [8]. This method is based on the analysis of the eigenvalues and eigenvectors of the signal's correlation matrix [9].

The main advantage of this algorithm is the ability to determine the frequencies and levels (amplitude and power) of the harmonic components (in addition to ability to obtain the spectrum as it is) [10].

The resulting dependence of the signal level on the frequency is called as the pseudospectrum. In addition, the parameter setting of MUSIC allows to use it as a filter with tunable sensitivity (it determines by the order of spectral transformation) to components of the signal's harmonics.

During the numerical simulation we have carried out the search process for the parameters of MUSIC algorithm that allow to identify the valve's failure in suction or pressurization lines. In order to calculate the pseudo-spectrum we used the function pmusic(). In order to calculate the frequencies and power of harmonics in the spectrum we used the rootmusic()-function from the libraries of Signal Processing Toolbox of the MathWorks Matlab ${ }^{\text {TM }}$ package [11].

\section{Results}

Our numerical investigations showed the possibility to estimate the degree of defect's evolution for various valve's defects in the compressor of NOGG (namely, the NOGG-70M) using the parameters of pseudo-spectra obtained by the MUSIC algorithm. The main parameters that correspond to defects in the valves (due to its aging or breakage) are: valve's spring stiffness, the flow section area, the response time and the weight of the valve.

We obtained the fact that the lowering of the order of transform (reduction of the number of approximating harmonics) up to 10-20 allows to get the spectrum without the components that correspond to high-frequency noise in the signal. It should also 
be pointed out that for the considered simulations of defects the parameters of the spectra of vibration velocity for the pressure under the piston are most sensitive (see the fig. 1).

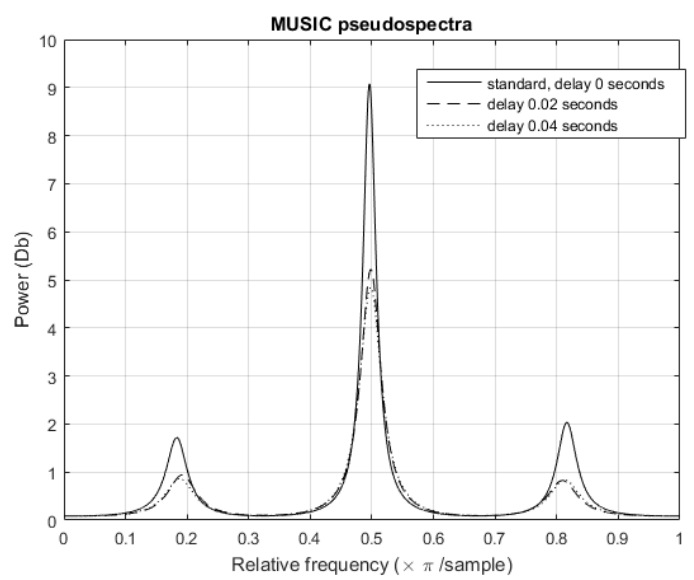

Fig. 1. Pseudo-spectra of vibration velocity for pressure under compressor's piston obtained by MUSIC algorithm

\section{References}

1. Plastinin PI. Piston compressors, vol. 1. Theory and calculations. Moscow: "Koloss" Publisher, 2006. [In Russian]

2. Kostyukov VN, Naumenko AP. Vibrodiagnostics of Piston Compressors. Compressors and Pneumatics, 2002; 3: 30-31. [In Russian]

3. Fillipov IV. Research of vibration processes in oil-injected screw compressors. Science and Education of Bauman MSTU, 2014; 10: 60-69. [In Russian]

4. Zhuravlyov OA, Komarov SYu, Popov KN, Prokofyev AB. Development of the automated method of vibration characteristics of power plants. Computer Optics, 2001; 21: 143149. [In Russian]

5. Grigoryev VA. Industrial power and thermal engineering. reference book. Moscow: "Energoatomizdat" Publisher, 1991. [In Russian]

6. Kondratyev TF. The valves of piston compressors. Leningrad: "Mashinostroenie" Publisher, 1983. [In Russian]

7. Dodin YuS, Klochkov VI, Lukyanica AI. The valves of piston compressors. Novomoskovsk: D. Mendeleev University of Chemical Technology of Russia, 2009. [In Russian]

8. Sergienko AB. Digital Signal Processing. St. Petersburg: "Piter" Pulisher, 2007. [In Russian]

9. Jian X, Zhang C, Zhao B, Zhu B. The application of MUSIC algorithm in spectrum reconstruction and interferogram processing. Optics Communications, 2008; 9: 2424-2428.

10. Cheney M. The Linear Sampling Method and the MUSIC Algorithm. Inverse Problems, 2001; 4(17).

11. MathWorks (official site). URL: http://matlab.ru/ (Date of inspect 02.03.2016). 